\title{
Wi-Fi Influence on LTE Downlink Data and Control Channel Performance in Shared Frequency Bands
}

\author{
Jiri MILOS, Ladislav POLAK, Stanislav HANUS, Tomas KRATOCHVIL \\ Dept. of Radio Engineering, Brno University of Technology, Technická 3082/12, 61600 Brno, Czech Republic \\ \{milos, polakl, hanus, kratot\}@feec.vutbr.cz
}

Submitted February 23, 2016 / Accepted December, 28, 2016

\begin{abstract}
Nowadays, providers of wireless services try to find appropriate ways to increase user data throughput mainly for future $5 G$ cellular networks. Utilizing the unlicensed spectrum (ISM bands) for such purpose is a promising solution: unlicensed frequency bands can be used as a complementary data pipeline for UMTS LTE (Universal Mobile Telecommunication System - Long Term Evolution) and its advanced version LTE-Advanced, especially in pico- or femtocells. However, coexisting LTE and WLAN services in shared ISM bands at the same time can suffer unwanted performance degradation. This paper focuses predominantly on co-channel coexistence issues (worst case) between LTE and WLAN (IEEE 802.11n) services in the ISM band. From the viewpoint of novelty, the main outcomes of this article are follows. Firstly, an appropriate signal processing approach for coexisting signals with different features in the baseband is proposed. It is applied in advanced link-layer simulators and its correctness is verified by various simulations. Secondly, the influence of IEEE 802.11n on LTE data and control channel performance is explored. Performance evaluation is based on error rate curves, depending on Signal-to-Interference ratio (SIR). Presented results allow for better understanding the influence of IEEE 802.11n on the LTE downlink physical control channels (PCCH) and are valuable for mobile infrastructure vendors and operators to optimize system parameters.
\end{abstract}

\section{Keywords}

LTE, WLAN, LTE physical channels, coexistence, interference, ISM band, $5 \mathrm{G}$

\section{Introduction}

The increasing demand high mobile data rates and the growing number of user equipments (e.g. Internet-of-Things services) brings forth the question of how to improve the performance or extend the functionality of existing $3 \mathrm{G} / 4 \mathrm{G}$ cellular networks, mainly in small cells. The licensed spectrum is the first choice for mobile operators thanks to its predictable behavior ensuring Quality of Service (QoS), mobility and system control. Naturally, the amount of available licensed spectrum is limited. Some former television bands have been sold to mobile operators in vendue. These frequency bands are almost fully occupied, especially in Europe [1-3]. Thus, there is a need for further expansion or a different solution.

Currently, the companies Qualcomm and Huawei are driving innovation to transfer Long Term Evolution (LTE) technology and its advanced version (LTE-A) to IndustryScience-Medical (ISM) unlicensed bands [4-6]. This concept is also called LTE-Unlicensed (LTE-U). Such innovation has been planned as a complementary or supporting data pipeline in small cells where demands on user data are higher. Both above mentioned companies have utilized the 2.4 GHz and $5 \mathrm{GHz}$ ISM bands for LTE/LTE-A and take advantage of respective signal propagation possibilities. The considered frequency bands are used especially for Wireless and Personal Local Area Network (WLAN and WPAN). Some mobile operators are building picocells and utilizing Wireless-Fidelity (Wi-Fi) networks in the 2.4 and $5 \mathrm{GHz}$ radio frequency (RF) bands as a supporting data pipeline in city centers or places with high density of user equipments [7], [8]. These Wi-Fi networks are controlled centrally by mobile operators. Locally, they have the potential to provide higher data throughput than $3 \mathrm{G} / 4 \mathrm{G}$ small cells.

Several recent papers deal with the study of coexistence of LTE and WLAN/WPAN networks in ISM bands [9]. Abinader et al. in [10] presented average user throughput results for coexisting LTE and Wi-Fi in indoor environment in the $5.8 \mathrm{GHz}$ unlicensed band and introduced a generalized collaborative coexistence algorithm. The presented results show that the average LTE user throughput value is similar to the case of non-coexistence scenarios and contrasts with degraded Wi-Fi average user throughput (for high Access Point (AP) density). Cavalcante in [11] provided a coexistence analysis of LTE and Wi-Fi in the $900 \mathrm{MHz}$ RF band under TGah indoor environment for an LTE system with system bandwidth of $20 \mathrm{MHz}$. Other studies [12-15] explored possible LTE average user throughput and performance decreasing under different LTE and Wi-Fi coexistence scenarios, mainly in $5 \mathrm{GHz}$ unlicensed bands. Appropriate simulation tools and methods for study of LTE and WLAN coexistence play a key role. A simulation-based study of the effect of LTE interference on an IEEE 802.11a system has been briefly discussed 


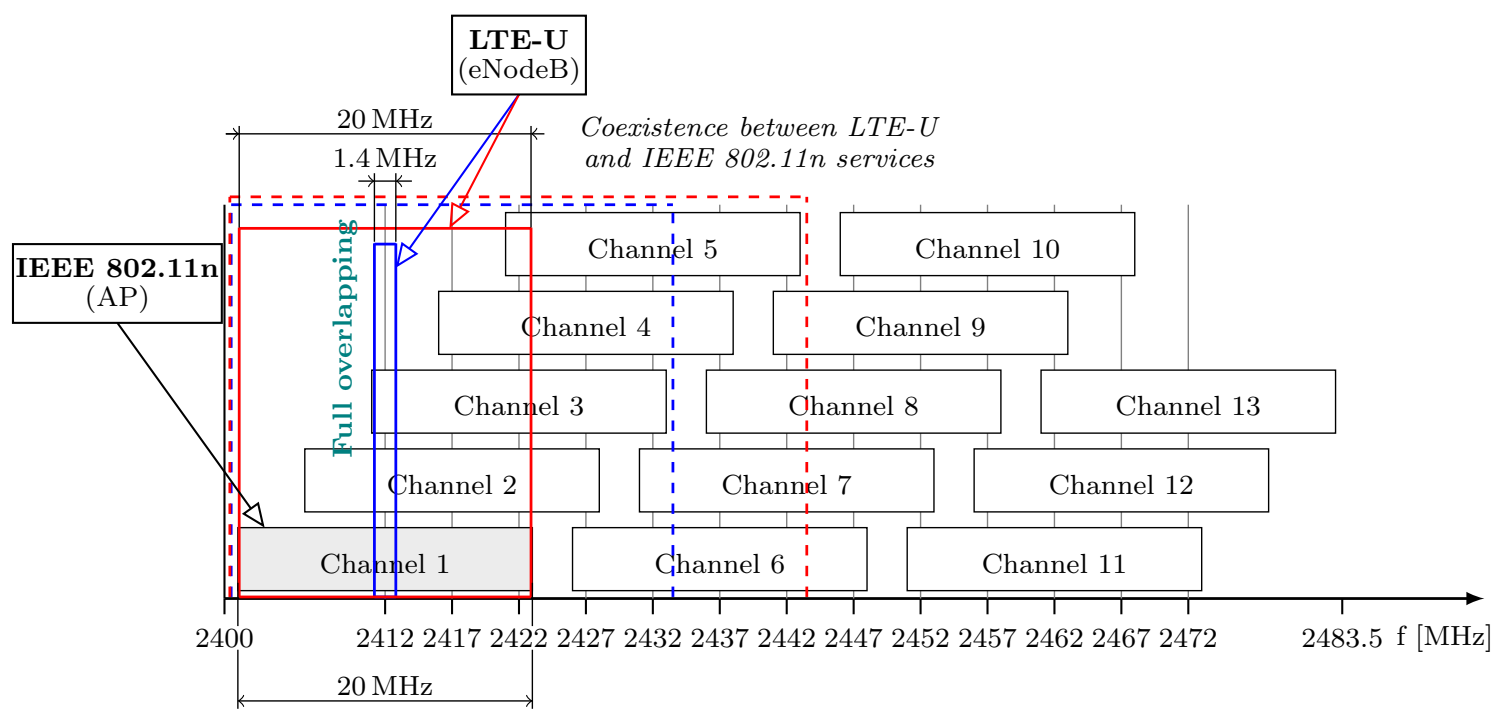

Fig. 1. Graphical representation of LTE-Unlicensed (1.4 and $20 \mathrm{MHz}$ system bandwidth marked by blue and red color, respectively) and Wi-Fi (IEEE 802.11n, system bandwidth $20 \mathrm{MHz}$ ) services in frequency domain, full overlapping in ISM $2.4 \mathrm{GHz}$ band.

in [16]. Comprehensive evaluation of the Licensed-Assisted Access (LAA) LTE and IEEE 802.11 coexistence scenarios based on system-level simulations were presented in [17]. In [18] the discrete-event Established Network Simulator (ns3) has been extended to study LTE and Wi-Fi coexistence in $5 \mathrm{GHz}$ band.

From this state-of-the-art review is evident that performance degradation of LTE and Wi-Fi communication systems caused by their coexistence on link-level has not been investigated in details. Hence, in this work we present an approach to analyse LTE including LTE downlink physical control channel (PCCH) performance at the link level influenced by Wi-Fi (based on IEEE 802.11n) in the same ISM frequency bands. To the best of our knowledge, no similar exploration in this form has been presented yet. The proposed methodology is appropriate especially for worst-case coexistence scenarios.

The main contributions of the article are follows:

1) An appropriate approach to emulate and evaluate coexistence between LTE (downlink) and Wi-Fi (IEEE $802.11 \mathrm{n}$ ) at the link-level in ISM bands is proposed.

2) Based on LTE and Wi-Fi co-channel coexistence evaluation, general conclusions for non-critical and critical coexistence scenarios are formulated.

The remaining part of this paper is composed as follows. The considered co-channel coexistence scenario between LTE and Wi-Fi is outlined in Section 2. Furthermore, Section 2 presents the proposed signal processing of coexisting LTE and Wi-Fi signals and its implementation to simulators. Section 3 states and discusses the simulated performance results of LTE (downlink) under the presented coexistence scenario. Finally, the main outputs of the work are clearly summarized in Conclusion.

\section{Coexistence Scenario}

Integration with the licensed spectrum, minimum changes in LTE air-interface and ensuring coexistence in unlicensed bands [19] are general design principles and prerequisites for LTE-U. Aggregation of licensed and unlicensed carriers brings better network performance, longer range and increasing capacity. Furthermore, unification of the LTE network with common authentication, security and management is an advantage. There are two main approaches for LTE-U. First one is supplemental downlink (SDL) which increases throughput only in downlink (main option for LTE Frequency Division Duplex (FDD)) whereas second one is carrier aggregation (CA) which increases throughput in both downlink and uplink (an option for LTE Time Division Duplex (TDD)). According to Huawei and its concept for unlicensed secondary carrier design for FDD [19], the following option is adopted: the primary cell (Pcell) FDD in downlink (user data+control information) is transmitted in the licensed band and the secondary cell (Scell) FDD in downlink (user data+control information) is transmitted in the unlicensed band. In this work, we only consider SDL due to the major use of LTE-FDD in the European region.

Currently, the $2.4 \mathrm{GHz}$ and $5 \mathrm{GHz}$ ISM bands are utilized by WLAN (Wi-Fi) and WPAN (e.g. Bluetooth, ZigBee) technologies. Wi-Fi, built on IEEE 802.11n and IEEE $802.11 \mathrm{ac}$ standards, is the dominant system in the $2.4 \mathrm{GHz}$ and $5 \mathrm{GHz}$ ISM bands, respectively [20]. Hence, modeling, measurement and suppression of interferences from mutual coexistence between WLAN/WPAN and LTE in ISM bands is becoming a key issue in future $5 \mathrm{G}$ networks. In Fig. 1, we can see the graphical representation of LTE-U and Wi-Fi services overlapping in the $2.4 \mathrm{GHz}$ ISM band. All possible WLAN channels with system bandwidth $B W_{\text {sys }}^{\mathrm{Wi}-\mathrm{Fi}}=20 \mathrm{MHz}$ are depicted with marked central frequencies. 


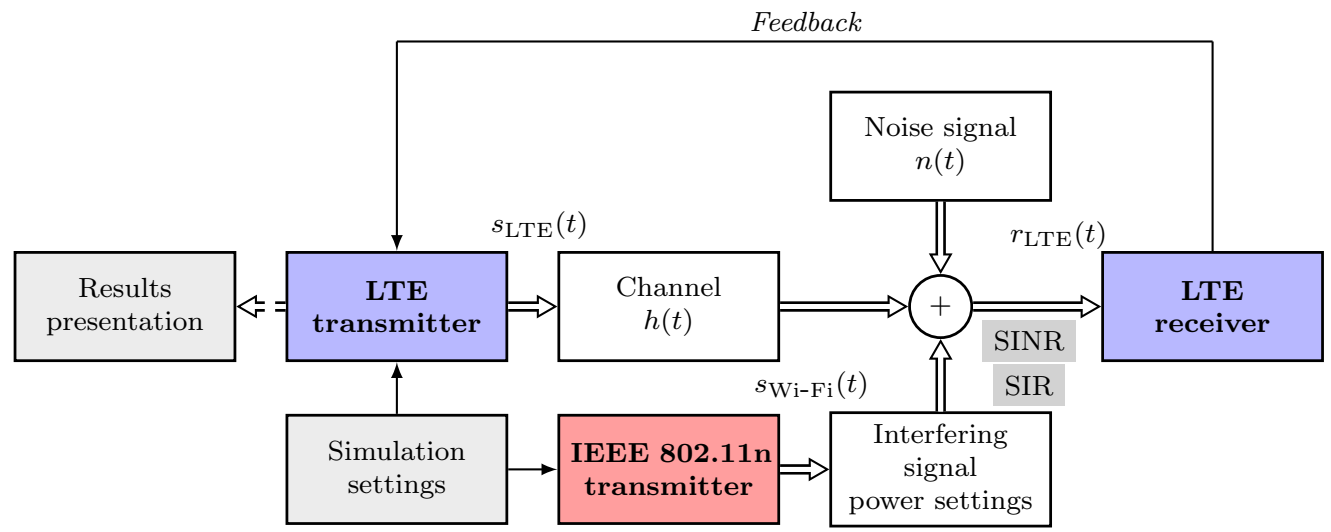

Fig. 2. Block diagram of the proposed LTE vs. IEEE $802.11 \mathrm{n}$ coexistence link-level simulator.

\begin{tabular}{|c|c|c|}
\hline Band [GHz] & Frequency range [MHz] & Bandwidth [MHz] \\
\hline \hline 2.4 & $2400-2483.5$ & 83.5 \\
\hline 5.1 & $5150-5350$ & 200 \\
\hline 5.3 & $5350-5470$ & 120 \\
\hline 5.4 & $5470-5825$ & 355 \\
\hline 5.8 & $5725-5875$ & 150 \\
\hline 5.9 & $5850-5925$ & 75 \\
\hline
\end{tabular}

Tab. 1. Summary of worldwide available $2.4 \mathrm{GHz}$ and $5 \mathrm{GHz}$ ISM frequency bands.

We consider that LTE services are provided on the first channel central frequency $\left(f_{\mathrm{c}}=2412 \mathrm{MHz}\right)$. LTE with $B W_{\text {sys }}^{\mathrm{LTE}}=1.4 \mathrm{MHz}$ is fully overlapped by Wi-Fi channels 1 , 2 and 3 whereas, LTE with $B W_{\text {sys }}^{\mathrm{LTE}}=20 \mathrm{MHz}$ is fully overlapping with Wi-Fi channel 1 and partially overlapping with channels 2, 3, 4 and 5 (see Fig. 1). According to the described scenario, we consider only co-channel inter-system coexistence (CIC) which represents the worst-case scenario. In such scenario the RF spectrum of one communication system is completely overlapping with the RF spectrum of other communication system (central frequencies of both systems are the same). Consequently, mutual interference of the systems is the greatest. It is equivalent to overlapping of the baseband signals [25]. A list of available unlicensed frequency bands is summarized in Tab. 1.

\subsection{Coexistence Link Level Simulator}

A block diagram of the proposed LTE-U/WLAN link level coexistence analysis model in downlink direction is depicted in Fig. 2. The LTE downlink link level simulator, developed at TU Vienna, was adopted as the basic simulation tool [26], [27]. We extended this MATLAB-based simulator by adding physical downlink control channel models. Thus, the impact of interferences from coexsitence between LTE and Wi-Fi is explored for all LTE downlink physical control channels, namely: Physical Control Format Indicator Channel (PCFICH), Physical Downlink Control Channel $(\mathrm{PDCCH})$ and Physical Hybrid ARQ Control Channel (PHICH) [28].

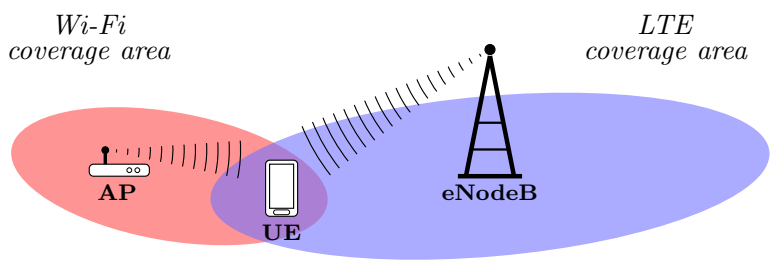

Fig. 3. Graphical representation of the LTE and Wi-Fi coexistence scenario in the same geographical area.

LTE control channels are used for signaling and transferring system information from the base station to individual user equipment (e.g. HARQ processing, user equipment power level settings, type of precoding) [29]. For emulation of the interfering Wi-Fi signal, we have proposed a universal WLAN link-level simulator supporting IEEE 802.11n technology. More details about this simulator can be found in [30].

Transceiver blocks in LTE downlink are marked with blue color and the interfering IEEE 802.11n system is marked with red color. The simulator was adjusted for simulations of inter-system coexistence. Motivated by processing time constraints, the simulator is implemented in baseband only, working with a complex envelope of the signal $s(t)=s_{\mathrm{I}}(t)+\mathrm{j} s_{\mathrm{Q}}(t)$, where $s_{\mathrm{I}}$ and $s_{\mathrm{Q}}$ are in-phase and quadrature components, respectively [31]. In this case, baseband signals in the frequency domain have a frequency spectra concentrated near zero frequency, which fits for the presented co-channel coexistence scenario. A simple graphical representation of the investigated LTE-U and Wi-Fi collision scenario in the same geographical area is shown in Fig. 3.

\section{Coexistence Channel Model}

The presented LTE vs. IEEE 802.11 n coexistence analysis model is based on general coexistence model described in time domain by the following equation:

$$
r_{0}(t)=\underbrace{s_{0}(t) * h_{0}(t)}_{\text {useful signal }}+\underbrace{n_{0}(t)}_{\text {noise }}+\underbrace{\sum_{k=1}^{N_{\mathrm{I}}}\left(s_{k}(t) * h_{k}(t)\right)}_{\text {interfering transmitters }}
$$


where $r_{0}(t)$ is the received useful signal, $s_{0}(t)$ is the transmitted useful signal, $h_{0}(t)$ is the impulse response of the useful channel (LTE branch), * represents discrete convolution and $n_{0}(t)$ is an additive white Gaussian noise added to the investigated receiver input. Interfering branch $s_{k}(t)$ is modeled as a sum of the signals from $N_{\mathrm{I}}$ interfering transmitters. Each interfering signal is led through a fading channel described by channel impulse response.

According to the presented coexistence scenario (see Fig. 2), the number of interferers $N_{\mathrm{I}}=1$ (IEEE 802.11n) and $h_{1}(t)=\delta(t)$ (delta function, no fading channel in the interfering branch). Thus we can rewrite (1) to

$$
r_{\mathrm{LTE}}(t)=\underbrace{s_{\mathrm{LTE}}(t) * h(t)}_{\text {LTE signal }}+\underbrace{n(t)}_{\text {noise }}+\underbrace{s_{\mathrm{Wi}-\mathrm{Fi}}(t)}_{\text {Wi-Fi signal }}
$$

where $r_{\mathrm{LTE}}(t)$ is the received LTE signal, $s_{\mathrm{LTE}}(t)$ is the transmitted LTE signal and $s_{\mathrm{Wi}-\mathrm{Fi}}(t)$ is the interfering Wi-Fi signal. The channel block adjusts the LTE output signal $s_{\text {LTE }}(t)$ in accordance with the used channel model and its impulse response $h(t)$. In this paper, no fading channel model is considered, thus $h(t)=\delta(t)$. Hence, (2) could be simplified to

$$
r_{\mathrm{LTE}}(t)=s_{\mathrm{LTE}}(t)+n(t)+s_{\mathrm{Wi}-\mathrm{Fi}}(t)
$$

The output signal $\left(s_{\mathrm{LTE}}(t)\right)$ from the LTE transmitter block (victim), is an OFDMA-based baseband signal in time domain with average power per symbol $\sigma_{s_{\mathrm{LTE}}(t)}^{2}=1$. The output signal from IEEE 802.11n (Wi-Fi, interferer) transmitter is an OFDM-based baseband signal in time domain $s_{\mathrm{Wi}-\mathrm{Fi}}(t)$ with average power per symbol $\sigma_{s_{\mathrm{Wi}-\mathrm{Fi}}(t)}^{2}=1$. After that, the $s_{\mathrm{LTE}}(t)$ signal passes through the channel model block, it enters the signal adder (see Fig. 2). Here, a random noise vector $n(t)$ and the interfering signal $s_{\mathrm{Wi}-\mathrm{Fi}}(t)$ are added to the useful signal $s_{\mathrm{LTE}}(t)$ according to the defined Signal-to-Interference plus Noise Ratio (SINR) and Signalto-Interference Ratio (SIR), respectively. In the presented scenario, SINR for each sample is defined as

$$
\mathrm{SINR}=\frac{P_{\mathrm{tx}}^{(\mathrm{LTE})}}{\sigma_{n}^{2}+P_{\mathrm{tx}}^{(\mathrm{Wi}-\mathrm{Fi})}}
$$

where $\sigma_{n}^{2}$ is the average power of the noise signal $n(t)$ which is modeled as a vector of normally-distributed random values with zero mean $\mu_{n(t)}=0, P_{\mathrm{tx}}^{(\mathrm{LTE})}$ is the power of LTE signal at the transmitter output and $P_{\mathrm{tx}}^{(\mathrm{Wi}-\mathrm{Fi})}$ is the power of $\mathrm{Wi}-\mathrm{Fi}$ signal in transmitter output. In the coexistence scenario considered, $\sigma_{n}^{2}=0$; hence, the SINR from (4) simply leads to SIR

$$
\mathrm{SIR}=\frac{P_{\mathrm{tx}}^{(\mathrm{LTE})}}{P_{\mathrm{tx}}^{(\mathrm{Wi}-\mathrm{Fi})}}
$$

Both SINR and SIR are defined as the post-FFT ratio at the receiving antenna (after FFT operation in the LTE receiver) [26]. Each sample of the received signal $r_{\text {LTE }}$ influenced by interfering singal is calculated as

$$
r_{\mathrm{LTE}}=s_{\mathrm{LTE}}+\frac{1}{\sqrt{2}} \frac{N_{\mathrm{FFT}}}{N_{\mathrm{tot}}} s_{\mathrm{Wi}-\mathrm{Fi}} 10^{\left(\frac{-\mathrm{SIR}_{(\mathrm{dB})}}{20}\right)}
$$

where $N_{\text {FFT }}$ is the FFT size used in LTE transmitter/receiver and $N_{\text {tot }}$ is the total number of subcarriers in a single LTE OFDMA symbol. In case of non-zero noise signal average power $\sigma_{n}^{2}$, the received signal $r_{\text {LTE }}$ definition is obtained by adding noise sample to (6), i.e.,

$r_{\mathrm{LTE}}=s_{\mathrm{LTE}}+\frac{1}{\sqrt{2}} \frac{N_{\mathrm{FFT}}}{N_{\mathrm{tot}}}\left[s_{\mathrm{Wi}-\mathrm{Fi}} 10^{\left(\frac{-\mathrm{SIR}_{\mathrm{dB}}}{20}\right)}+n 10^{\left(\frac{-\mathrm{SNR}_{\mathrm{ch}(\mathrm{dB})}}{20}\right)}\right]$

where $\mathrm{SNR}_{\mathrm{ch}}$ is the SNR in considered AWGN channel model $\left(\mathrm{SNR}_{\mathrm{ch}}=P_{\mathrm{tx}}^{(\mathrm{LTE})} / \sigma_{n}^{2}\right)$ with constant value in whole simulation. In case of added AWGN (7), SINR defined in (4) is an independent value in the coexistence model and numerically SINR $\approx$ SIR. As mentioned above, two baseband signals (LTE and Wi-Fi) are added due to the property of linearity considered for this scenario. Interpolation is not used since it has negligible influence to performance results [35].

The interfered LTE signal $r_{\mathrm{LTE}}(t)$ with additive noise enters the LTE receiver, where inverse OFDMA operations are performed. According to the used SISO transmission mode, the multi-antenna decoding process is not necessary. Due to using the AWGN channel model, an estimation of used transmission channel is not provided here, thus the LTE receiver has perfect knowledge of the channel. Furthermore, Soft-Sphere Decision (SSD) is used as the demodulation algorithm [26]. We also assume a static transmission scenario (no movement of LTE receiver).

In the presented LTE downlink simulator, the physical control channel (PCFICH, PHICH and PDCCH) signal processing at the receiving side is performed separately from the physical downlink shared channel (PDSCH) which carries user data [29]. Channel decoding and evaluation of the received information is performed. The received user data, control information, data acknowledgement information and Channel Quality Indicator (CQI) are reported to the LTE transmitter block. Of course, the influenced signal $r(t)$ could also lead to the IEEE 802.11n receiver where inverse OFDM operations are performed and the obtained results are evaluated [30].

\section{Physical Downlink Shared Channel}

Quality of reception of user data in LTE downlink transmitted via PDSCH depends mainly on quality of the respective transmission channel, used modulation and channel coding, the use of Hybrid-ARQ (HARQ) retransmissions and link adaptation algorithms and user data scheduling. The modulation and channel coding scheme used in PDSCH is determined from CQI parameter. The list of these parameters is presented in Tab. 2. The CQI index [26] defines the corresponding modulation type and code rate, including channel coding efficiency. Due to only having a single user in the simulation scenario, there is no demand for user data scheduling (user data occupies all available resource elements (REs)). In the presented simulations, CQI is always defined as fixed. 


\begin{tabular}{|c|c|c|c|}
\hline $\begin{array}{c}\text { CQI } \\
\text { index }\end{array}$ & Modulation & $\begin{array}{c}\text { Code rate } \\
\times \mathbf{1 0 2 4}\end{array}$ & Efficiency \\
\hline \hline 1 & & 78 & 0.1523 \\
2 & & 120 & 0.2344 \\
3 & & 193 & 0.3770 \\
4 & QPSK & 308 & 0.6016 \\
5 & & 449 & 0.8770 \\
6 & & 602 & 1.1758 \\
\hline 7 & & 378 & 1.4766 \\
8 & $16-Q A M$ & 490 & 1.9141 \\
9 & & 616 & 2.4063 \\
\hline 10 & & 466 & 2.7305 \\
11 & & 567 & 3.3223 \\
12 & \multirow{2}{*}{$64-\mathrm{QAM}$} & 666 & 3.9023 \\
13 & & 772 & 4.5234 \\
14 & & 873 & 5.1152 \\
15 & & 948 & 5.5547 \\
\hline
\end{tabular}

Tab. 2. The list of LTE Channel Quality Indicator (CQI) parameters [26].

\section{Physical Control Format Indicator Channel}

The Control Format Indicator (CFI) parameter is transmitted via PCFICH. CFI contains two-bit value which defines mapping of PDCCH in LTE downlink subframe [28]. PCFICH uses block channel coding with code rate $1 / 16$. PCFICH is always placed in the first OFDM symbol in downlink subframe. In the frequency domain, PCFICH is spread into four parts (additional frequency diversity) and its position is also given by cell identification number $N_{\text {cell }}^{\mathrm{ID}}$.

\section{Physical Downlink Control Channel}

PDCCH is the most important control channel in LTE downlink since it carries system information aboout scheduling grants, MIMO settings, modulation and channel coding (CQI) etc. PDCCH symbols are located at the beginning of each subframe while the number of OFDM symbols for $\mathrm{PDCCH}$ is defined by CFI value [28]. PDCCH uses convolutional coding with code rate $1 / 3$. At the receiver, the $\mathrm{PDCCH}$ is processed via combination of Blind and Viterbi decoding process.

\section{Physical Hybrid-ARQ Indicator Channel}

PHICH transmits the Hybrid-ARQ Indicator (HI) which contains acknowledge or non-acknowldge message of previous user data sent in uplink [29]. PHICH uses simple repetition channel coding with code rate $1 / 3$. Repeated symbols are spread using up to 8 orthogonal sequences. Number of PHICH transmitted in single subframe depends mainly on system bandwidth and PHICH scaling factor $N_{\mathrm{g}}$ which defines number of PHICH groups in single subframe. PHICH is situated either in the first or the first and second OFDM symbol. Each PHICH group is also spread into three parts. Their position is given by $N_{\text {cell }}^{\mathrm{ID}}$ as well [29].

\section{Coexistence Analysis}

LTE and Wi-Fi (IEEE 802.11n) general system parameters considered in the presented simulation scenario are sumarized in Tab. 3. For LTE we consider two extreme system bandwidths $B W_{\text {sys }}^{\mathrm{LTE}}=1.4 \mathrm{MHz}$ (narrowest) and $20 \mathrm{MHz}$ (widest) and subcarrier spacing $\Delta_{\mathrm{f}}^{\mathrm{LTE}}=15 \mathrm{kHz}$. The Wi-Fi system model is considered for system $B W_{\text {sys }}^{\mathrm{Wi}-\mathrm{Fi}}=20 \mathrm{MHz}$

\begin{tabular}{|c|c|c|c|c|c|}
\hline \multicolumn{3}{|c|}{ System parameter } & \multicolumn{2}{|c|}{ LTE } & Wi-Fi \\
\hline $\begin{array}{l}\text { System } \\
\text { bandwidth }\end{array}$ & $B W_{\text {sys }}$ & {$[\mathrm{MHz}]$} & 1.4 & 20 & 20 \\
\hline $\begin{array}{l}\text { Number of } \\
\text { occupied } \\
\text { subcarriers }\end{array}$ & $N_{\mathrm{sc}}$ & {$[-]$} & 72 & 1200 & 56 \\
\hline $\begin{array}{l}\text { IFFT/FFT } \\
\text { size }\end{array}$ & $N_{\mathrm{FFT}}$ & {$[-]$} & 128 & 2048 & 64 \\
\hline $\begin{array}{l}\text { Subcarrier } \\
\text { space }\end{array}$ & $\Delta_{\mathrm{f}}$ & {$[\mathrm{kHz}]$} & 15 & 15 & 312.5 \\
\hline
\end{tabular}

Tab. 3. General physical layer parameters of the LTE and Wi-Fi link-level models.

\begin{tabular}{|c|c|}
\hline Simulation parameter & Value \\
\hline Signal-to-Interference ratio (SIR) range & $\langle-30,30\rangle \mathrm{dB}$ \\
\hline Number of transmitted subframes & 2000 \\
\hline Channel Quality Indicator range & $\langle 1,15\rangle$ \\
\hline $\begin{array}{ll}\text { PDSCH: } & \begin{array}{l}\text { Number of allocated users } \\
\text { Scheduling algorithm }\end{array}\end{array}$ & $\begin{array}{c}1 \\
\text { static and fixed }\end{array}$ \\
\hline $\begin{array}{l}\text { Group scaling factor } \\
\text { HI (users) per group }\end{array}$ & $N_{\mathrm{g}}=\left\{\begin{array}{c}1 / 6,1 / 2,1,2\} \\
1\end{array}\right.$ \\
\hline CFI message range & $\langle 1,4\rangle$ or $\langle 0,3\rangle$ \\
\hline Transmitted DCI format & $\mathrm{F} 0$ \\
\hline
\end{tabular}

Tab. 4. Simulation parameters used in the presented link-level coexistence scenario.

only and $\Delta_{\mathrm{f}}^{\mathrm{Wi}-\mathrm{Fi}}=312.5 \mathrm{kHz}$. Extended Wi-Fi system bandwidth of $40 \mathrm{MHz}$ is not modeled.

The number of occupied subcarriers equals to 56 according to the definition of the Greenfield Wi-Fi mode [20]. We consider single-user SISO (SU-SISO) transmission mode for both systems. For LTE it means a single base station (eNodeB) and a single user equipment (UE).

Before the coexistence simulation starts, it is necessary to define simulation parameters. Parameters common to the LTE/Wi-Fi trnansmitter and LTE receiver are listed in Tab. 4. The independent SIR variable should be set together with the number of transmitted LTE subframes $\left(N_{\text {subf }}=2000\right)$. The $\mathrm{PDSCH}$ performance is simulated for CQI in range from 1 to 15 (see Tab. 2) as a fixed value. PHICH error rate performance is simulated for all available scaling factor $N_{\mathrm{g}}$ values with single user per $\mathrm{PHICH}$ group. The performance results (BER, BLER, throughput) in all physical channels are computed by averaging over all their allocated resource elements.

\subsection{Simulation Results}

In this section, results of the LTE vs. Wi-Fi coexistence analysis are presented and discussed. The LTE PDSCH link performance represented as a dependence of Block Error Rate (BLER) on SIR is shown in Fig. 4. Results were obtained for various CQI values and system bandwidths of 1.4 and $20 \mathrm{MHz}$. As we can see from presented BLER results, LTE receiver with CQI value set in the range from 13 to 15 is unable to reach the required 10\% BLER and thus, these $\mathrm{CQI}$ are unusable for user data transmission under presented LTE/WLAN coexistence scenario. The BLER reference level $10^{-1}=10 \%$ BLER was determined according to the required target quality for LTE shared physical channel user data reception [21]. 


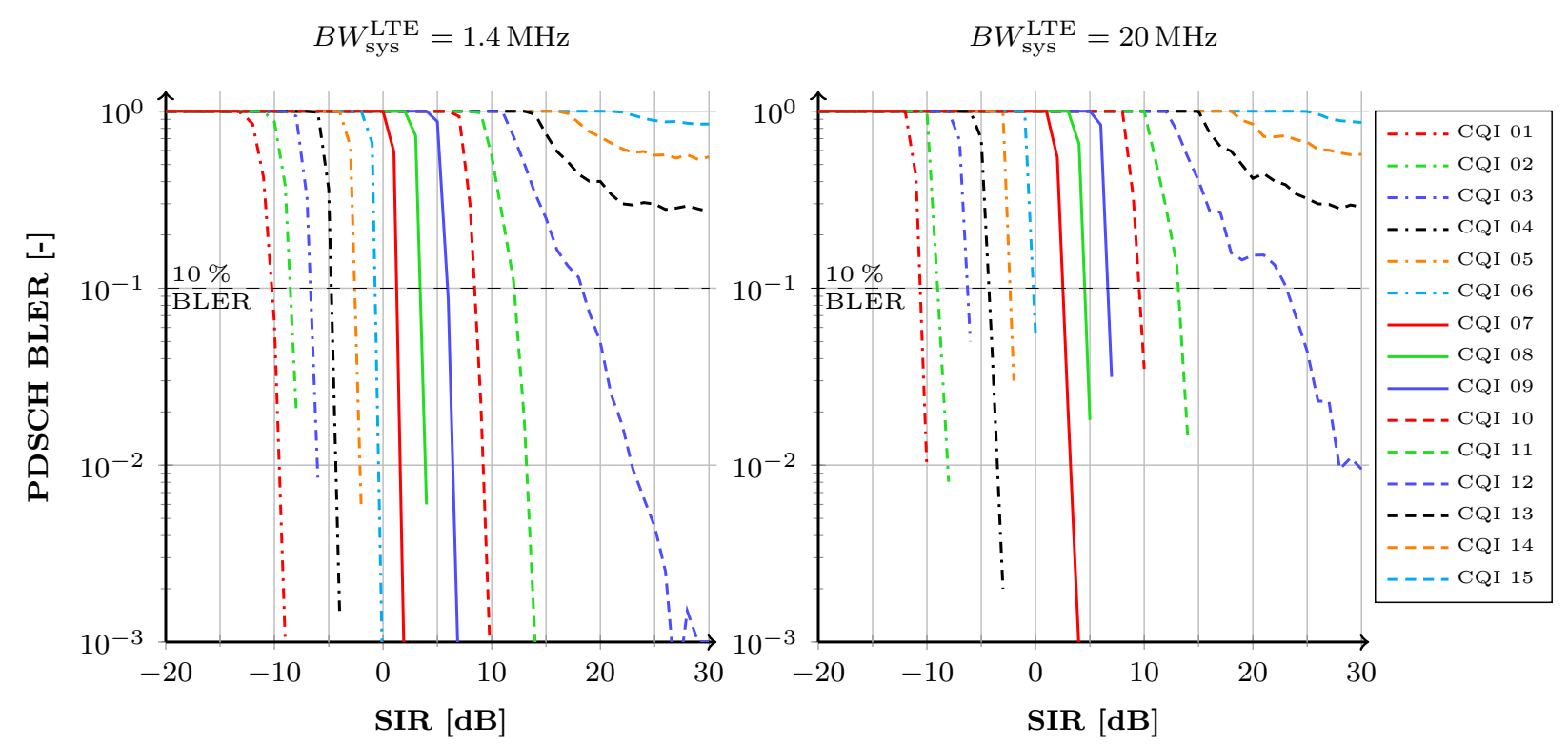

Fig. 4. LTE PDSCH Block error rate link performance for various CQI's under the Wi-Fi co-channel coexistence scenario $\left(B W_{\mathrm{sys}}^{\mathrm{LTE}}=1.4 \mathrm{MHz}\right.$ and $\left.20 \mathrm{MHz}, B W_{\text {sys }}^{\mathrm{Wi}-\mathrm{Fi}}=20 \mathrm{MHz}\right)$.

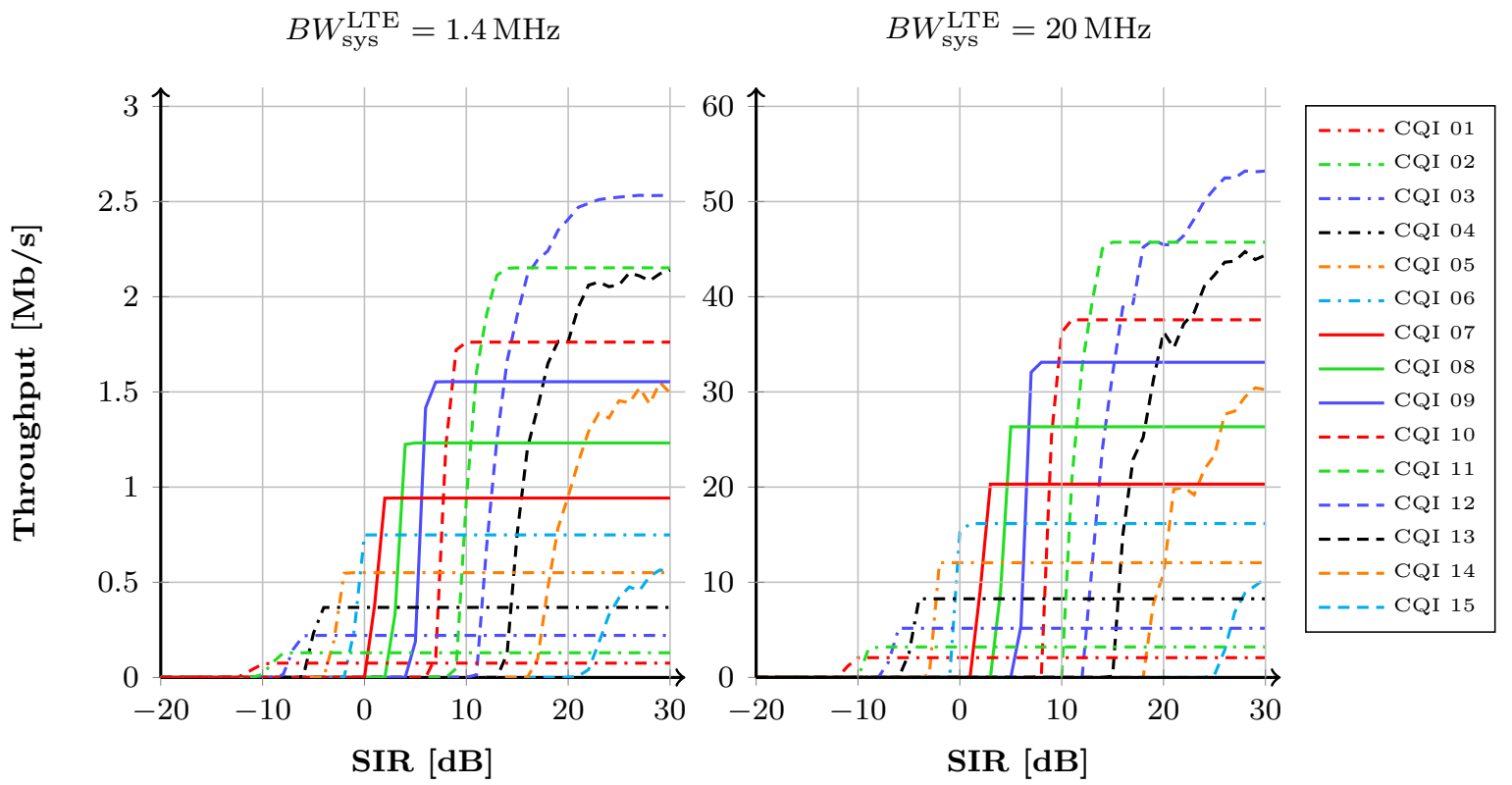

Fig. 5. LTE PDSCH user data throughput for various CQI's under the Wi-Fi co-channel coexistence scenario $\left(B W_{\text {sys }}^{\mathrm{LTE}}=1.4 \mathrm{MHz}\right.$ on the left, $B W_{\text {sys }}^{\mathrm{LTE}}=20 \mathrm{MHz}$ on the right), $N_{\text {subf }}=2000, B W_{\text {sys }}^{\mathrm{Wi}-\mathrm{Fi}}=20 \mathrm{MHz}$.

LTE PDSCH throughput curves versus SIR for $B W_{\text {sys }}^{\mathrm{LTE}}=1.4 \mathrm{MHz}$ and $20 \mathrm{MHz}$ are shown in Fig. 5. From the results it is seen that LTE throughput is measurable for SIR higher than $-12 \mathrm{~dB}$, independently of the considered LTE system bandwidth. Using of CQI $=13,14$ and 15 could bring unpredictable behavior. Using of $\mathrm{CQI}=12$ is possible but the throughput is slightly decreased compared to non-coexistence scenario results [27].

The LTE PDSCH to SIR mapping for $10 \%$ BLER is shown in Fig. 6. These mapping curves are very important for modeling and simulation methodology. This mapping is termed effective SINR/SIR mapping (ESM). It serves as the basis for physical layer abstraction for link-to-system level mapping, including MIMO technology [22], [23]. The concept is also used in Vienna LTE System-level simulator for LTE intra-system interference evaluation [24]. The CQI to SIR or SINR mapping is approximately linear in noncoexistence scenarios due to equidistant points of intersection [24]. Here, the equidistance is not obtained for CQI using 64QAM (10,11 and 12). As we can see from the figure, $\mathrm{CQI}=13,14$ and 15 are not mapped to SIR (these curves do not reach $10 \%$ BLER).

Figure 7 presents the BER performance results of the co-channel inter-system interference, depending on SIR, in the PCFICH and PDCCH physical control channel. PCFICH missdetection could bring packet loss and even short-term 
loss of connection. Hence, exploring of its performance is important. One percent PCFICH bit error rate (see Fig. 7 on the left $)$ is reached for $\mathrm{SIR}=-14.1 \mathrm{~dB}\left(B W_{\text {sys }}^{\mathrm{LTE}}=1.4 \mathrm{MHz}\right)$ and $-12.8 \mathrm{~dB}\left(B W_{\text {sys }}^{\mathrm{LTE}}=20 \mathrm{MHz}\right)$. $\mathrm{PDCCH}$ information is transmitted with one percent bit error at SIR $=0 \mathrm{~dB}$ (see Fig. 7 on the right). Evaluated results reveal the fact that the LTE system bandwidth used has negligible effect on the LTE PDCCH physical downlink control channel performance at its co-channel coexistence with IEEE 802.11n.

Finally, LTE PHICH control channel performances affected by co-channel Wi-Fi interferences was investigated and the obtained results described as BER vs. SIR dependence are shown in Fig. 8. The purpose PHICH channel in the downlink is to carry HARQ acknowledgements for uplink data transfers. PHICH performance requirements are defined by 3GPP [33]. PHICH BER (acknowledge to nonacknowledge error and vice versa) should be within the range from $10^{-3}$ to $10^{-4}$ (see gray filled area in Fig. 8). Firstly, PHICH performances were obtained for various scaling factors $\left(N_{\mathrm{g}}\right)$ at $B W_{\text {sys }}^{\mathrm{LTE}}=1.4 \mathrm{MHz}$. In such case (see blue curves in Fig. 8), parameter $\left(N_{\mathrm{g}}\right)$ has no influence on PHICH performance.

Mapping of LTE downlink physical control channels depends mainly on cell identification number $\left(N_{\text {cell }}^{\mathrm{ID}}\right)$, system bandwidth $B W_{\text {sys }}^{\mathrm{LTE}}$, PHICH scaling factor $N_{\mathrm{g}}$ and amount of system information to transmit (PDCCH). Example of the LTE downlink physical control channels mapping (symbols 1 and 2) for the highest system bandwidth $B W_{\text {sys }}^{\mathrm{LTE}}=20 \mathrm{MHz}$, $N_{\text {cell }}^{\mathrm{ID}}=0$ is depicted in Fig. 9. Here, the LTE spectrum is fully overlapping with Wi-Fi spectrum (IEEE 802.11n, $B W_{\text {sys }}^{\mathrm{Wi}-\mathrm{Fi}}=20 \mathrm{MHz}$ ). The part of bandwidth used for user data and control information transmitting ( $\left.B W_{\text {used }}\right)$ is usually less than the system bandwidth in OFDM-based systems. LTE with system bandwidth $20 \mathrm{MHz}$ uses 1200 subcarriers in frequency domain and $1 \mathrm{DC}$ subcarrier. Due to $\Delta_{\mathrm{f}}^{\mathrm{LTE}}=15 \mathrm{kHz}$ distance between subcarriers, the used bandwidth $B W_{\text {used }}^{\mathrm{LTE}}=(1200+1) \times 15 \mathrm{kHz}=18.015 \mathrm{MHz}$.

The Wi-Fi (IEEE 802.11n) with the same system bandwidth uses only 56 subcarriers and 1 DC subcarrier. The distance between subcarriers in Wi-Fi system is much higher $\left(\Delta_{\mathrm{f}}^{\mathrm{Wi}-\mathrm{Fi}}=312.5 \mathrm{kHz}\right)$, thus $B W_{\text {used }}^{\mathrm{Wi}-\mathrm{Fi}}=(56+1) \times 312.5 \mathrm{kHz}=$ $17.8125 \mathrm{MHz}$. There is $98.88 \%$ overlap in LTE spectrum. As we can see from Fig. 9, amount of PHICH influenced by Wi-Fi interference also depends on scaling factor $N_{\mathrm{g}}$, where a specific part of $\mathrm{PHICH}$ is not fully overlapped by Wi-Fi spectrum (see magnified image).

The same dependences were investigated for $B W_{\text {sys }}^{\mathrm{LTE}}=$ $20 \mathrm{MHz}$. At the widest LTE system bandwidth, there is a significant difference of $\mathrm{PHICH}$ performance for various $N_{\mathrm{g}}$ values. When $N_{\mathrm{g}}=1 / 6$ is considered then to fulfil BER range from $10^{-3}$ to $10^{-4}$ the coding gain for $B W_{\text {sys }}^{\mathrm{LTE}}=$ $20 \mathrm{MHz}$ is approximately $11 \mathrm{~dB}$ less in comparison with $B W_{\text {sys }}^{\mathrm{LTE}}=1.4 \mathrm{MHz}$. Such behaviour is given by different PHICH mapping and different amounts of interfered resource elements in the resource grid (see gray filled areas in Fig. 9).

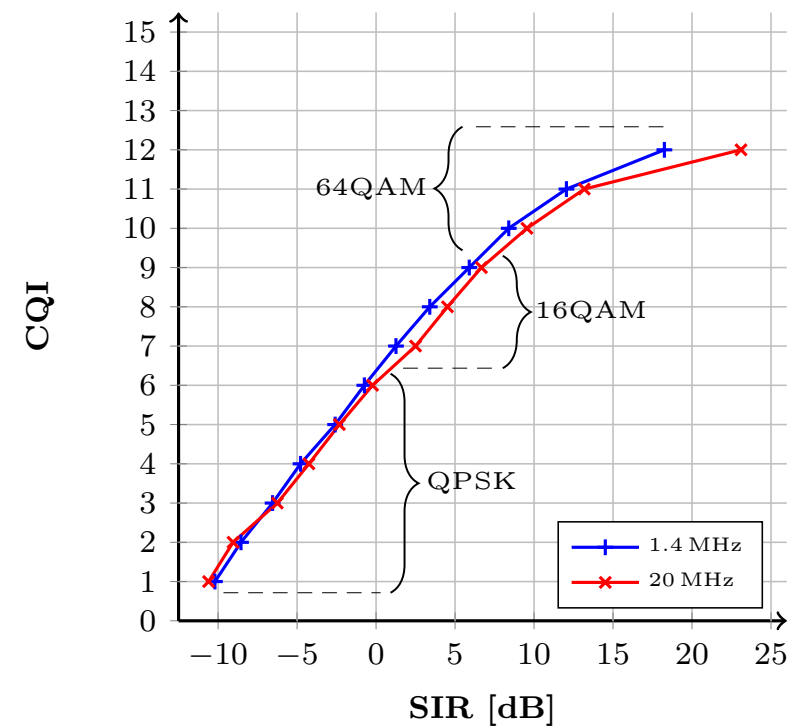

Fig. 6. LTE PDSCH CQI to SIR ESM mapping under the Wi-Fi co-channel coexistence scenario.
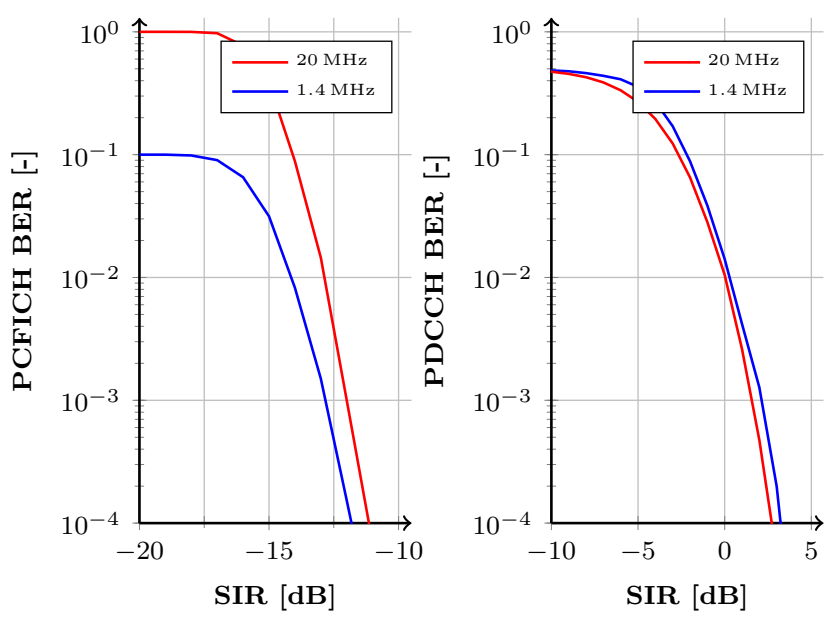

Fig. 7. LTE PCFICH and PDCCH control channel performance under the Wi-Fi co-channel coexistence scenario (PCFICH on the left, PDCCH on the right).

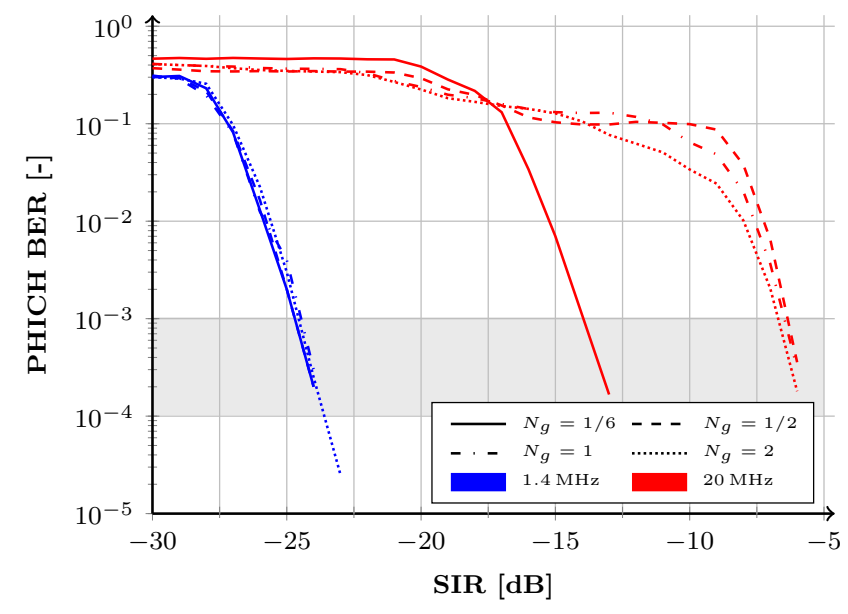

Fig. 8. LTE PHICH control channel performance for various scaling factors $N_{\mathrm{g}}$ under the Wi-Fi co-channel coexistence scenario $\left(B W_{\mathrm{sys}}^{\mathrm{LTE}}=1.4 \mathrm{MHz}\right.$ blue, $B W_{\mathrm{sys}}^{\mathrm{LTE}}=$ $20 \mathrm{MHz}$ red). 


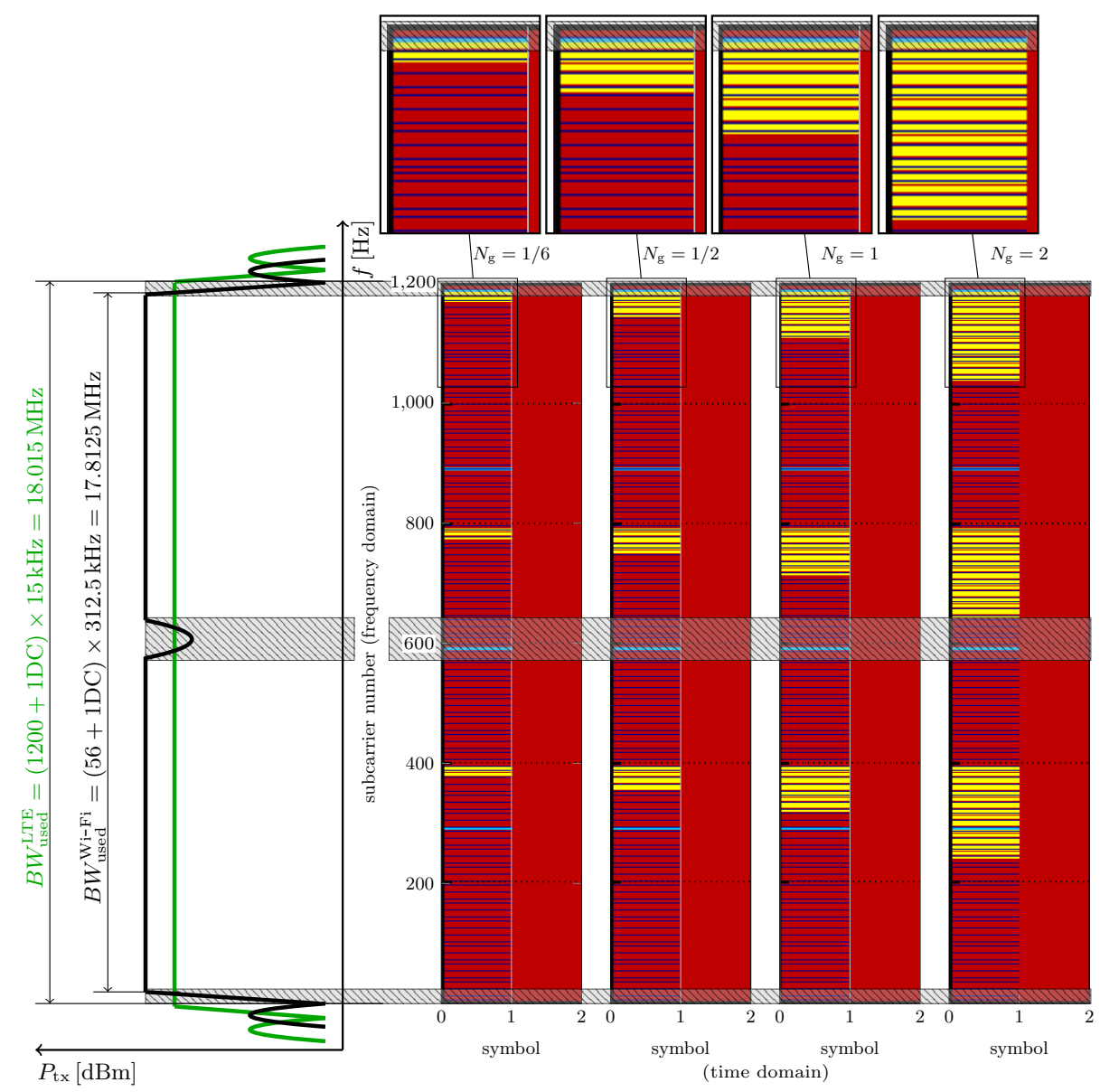

Fig. 9. $\mathrm{LTE}$ physical control channel mapping in resource grid $B W_{\mathrm{sys}}^{\mathrm{LTE}}=20 \mathrm{MHz}, N_{\text {cell }}^{\mathrm{ID}}=0$ (example case) and scaling factor $N_{\mathrm{g}}($ red $=$ PDCCH, blue $=$ PCFICH, yellow $=$ PHICH) and LTE spectrum (green) fully overlapped by a Wi-Fi spectrum (black, IEEE 802.11n, $B W_{\text {sys }}^{\mathrm{Wi}} \mathrm{Fi}=20 \mathrm{MHz}$ ) in frequency domain (on the left). Magnified part of the physical control channels mapping is on the top.

Overall, BER PHICH curves reach the reference value $10^{-3}$ at $\mathrm{SIR}=-24.6 \mathrm{~dB}$ for $B W_{\text {sys }}^{\mathrm{LTE}}=1.4 \mathrm{MHz}, \mathrm{SIR}=-14 \mathrm{~dB}$ for $B W_{\text {sys }}^{\mathrm{LTE}}=20 \mathrm{MHz}, N_{\mathrm{g}}=1 / 6$ and $\mathrm{SIR}=-6.6 \mathrm{~dB}$ for $B W_{\text {sys }}^{\mathrm{LTE}}=20 \mathrm{MHz}, N_{\mathrm{g}} \neq 1 / 6$. From these values (lower SIR) is evident that LTE PHICH downlink channel has good resistance features against interferences from coexistence with Wi-Fi.

\section{Conclusion}

This paper introduces a novel approach in analysing LTE link-level coexistence issues in shared frequency bands. It presents the results of LTE data and control channel performance under co-channel inter-system coexistence with WLAN (Wi-Fi) services. For the analysis, the LTE-U vs. Wi-Fi coexistence link level simulator was created. The presented simulator could provide a basic tool for research in the field of controlled heterogenous networks and cooperative algorithms. The analysis of the obtained results from the presented co-channel coexistence scenario leads to the following general conclusions:

1. LTE transmission is robust and resistant against interference from Wi-Fi services (IEEE 802.11n) in shared frequency bands.
2. User data transmitted via LTE PDSCH are well protected for the link with lower CQI index (from 1 to 11). PDSCH link with CQI = 12 operates properly for SIR greater than $15 \mathrm{~dB}$. Using the PDSCH link with CQI higher than 12 is not suitable for LTE vs. Wi-Fi (IEEE 802.11n) co-channel coexistence scenarios.

3. LTE PDSCH CQI to SIR ESM mapping is linear in the range of CQI from 1 to 9 and non-linear in the range of CQI from 10 to 12 (CQI $=13,14$ and 15 are unusable for transmission in the LTE vs. WLAN coexistence scenario).

4. Scalable bandwidth in LTE has inconsiderable impact on the PDCCH physical control channel performance (convolutional channel coding with code rate 1/3). The performance of PCFICH is more influenced by scalable bandwidth, where $\triangle \mathrm{SIR} \approx 1.8 \mathrm{~dB}$ at $\mathrm{PCFICH}$ BER equaling to $10^{-2}$ (block channel coding with code rate $1 / 16)$.

5. For the presented scenario, the scaling factor $N_{\mathrm{g}}$ highly affects PHICH control channel performance mainly for LTE system bandwidth $20 \mathrm{MHz}$. The PHICH BER results for $N_{\mathrm{g}}=1 / 6$ show a gain $7 \mathrm{~dB}$ (at PHICH BER $10^{-3}$ ) comparing to other $N_{\mathrm{g}}$ values. 
Coexistence methodology and results presented in this paper enable to better understand the LTE performance and reliability at the same frequency bands and location area with WLAN. This paper defines conditions and preliminary recommendations for LTE and Wi-Fi operation of uncontrolled LTE-U networks. The results could be valuable for developers, vendors and mobile operators to optimize system parameters.

\section{Acknowledgments}

This work is supported by CATRENE under the project named CORTIF CA116, the MEYS of the Czech Republic no. LF14033 and by the BUT project no. FEKTS-14-2177 (PEKOS). The described research was performed in laboratories supported by the SIX project; no. CZ.1.05/2.1.00/03.0072, the operational program Research and Development for Innovation. The research described in this paper was financed by the Czech Ministry of Education in the frame of the National Sustainability Program under grant LO1401. For the research, the infrastructure of the SIX Center was used.

\section{References}

[1] FUENTES, M., GARCIA-PARDO, C., GARRO, E., et al. Coexistence of digital terrestrial television and next generation cellular networks in the $700 \mathrm{MHz}$ band. IEEE Wireless Communications, 2014, vol. 21, no. 6, p. 63-69. DOI: 10.1109/MWC.2014.7000973

[2] POLAK, L., KALLER, O., KLOZAR, L., et al. Coexistence between DVB-T/T2 and LTE standards in common frequency bands. Wireless Personal Communications, 2016, vol. 88, no. 3, p. 669-684. DOI: $10.1007 / \mathrm{s} 11277-016-3191-2$

[3] POLAK, L., KLOZAR, L., KALlER, O., et al. Study of coexistence between indoor LTE femtocell and outdoor-to-indoor DVBT2-Lite reception in a shared frequency band. EURASIP Journal on Wireless Communications and Networking, 2015, no. 114, p. 1-14. DOI: $10.1186 / \mathrm{s} 13638-015-0338-\mathrm{x}$

[4] HUAWEI TECHNOLOGIES CO., LTD., CHINA. The Unlicensed Spectrum Usage for Future IMT Technologies (white paper). 18 pages. [Online] Cited 2015-05-15. Available at: http://www.huawei.com/

[5] QUALCOMM TECHNOLOGIES, INC., USA. Extending the benefits of LTE Advanced to unlicensed spectrum (white paper). 19 pages. [Online] Cited 2015-05-15. Available at: http://www.qualcomm.com/

[6] QUALCOMM TECHNOLOGIES, INC., USA. LTE in Unlicensed Spectrum: Harmonious Coexistence with Wi-Fi (white paper). 19 pages. [Online] Cited 2015-05-15. Available at: http://www.qualcomm.com/

[7] FUJITSU NETWORK COMMUNICATIONS INC., TEXAS, USA High-Capacity Indoor Wireless Solutions: Picocell or Femtocell (white paper). 10 pages. [Online] Cited 2015-05-15. Available at: http://www.us.fujitsu.com/telecom/

[8] 3rd GENERATION PARTNERSHIP PROJECT, TECHNICAL SPECIFICATION GROUP SERVICES AND SYSTEM ASPECTS, FRANCE Architecture enhancements for non-3GPP accesses (technical specification). 131 pages. [Online] Cited 2015-05-16. Available at: http://www.3gpp.org/DynaReport/23402.htm
[9] JOENGHO, J., HUANING, N., LI, Q. C., et al. LTE in the unlicensed spectrum: Evaluating coexistence mechanisms. In Proceedings of the Globecom Workshops. 2014, p. 740-745. DOI: 10.1109/GLOCOMW.2014.7063521

[10] ABINADER, F. M., ALMEIDA, E. P. L., CHAVES, F. S., et al Enabling the coexistence of LTE and Wi-Fi in unlicensed bands. IEEE Communications Magazine, 2014, vol. 52, no. 11, p. 54-61. DOI: 10.1109/MCOM.2014.6957143

[11] CAVAlCANTE, A. M., AlmEIDA, E., VIEIRA, R. D., et al. Performance evaluation of LTE and Wi-Fi coexistence in unlicensed bands. In Proceedings of the 77th IEEE Vehicular Technology Conference (VTC Spring). Jun. 2013, p. 1-6. DOI: 10.1109/VTCSpring.2013.6692702

[12] RONGRONG, S., XINGLIN, W. Analysis and test for co-site of LTE and WiFi system. In Proceedings of the IEEE International $R F$ and Microwave Conference (RFM). Dec. 2011, p. 315-319. DOI: 10.1109/RFM.2011.6168757

[13] BHORKAR, A., IBARS, C., PINGPING, Z. On the throughput analysis of LTE and WiFi in unlicensed band. In Proceedings of the 48th Asilomar Conference on Signals, Systems and Computers. Nov. 2014, p. 1309-1313. DOI: 10.1109/ACSSC.2014.7094671

[14] AL-DULAIMI, A., AL-RUBAYE, S., QIANG, N., et al. 5G communications race: Pursuit of more capacity triggers LTE in unlicensed band. IEEE Vehicular Technology Magazine, Mar. 2015, vol. 10, no. 1, p. 43-51. DOI: 10.1109/MVT.2014.2380631

[15] JOENGHO, J., LI, Q. C., HUANING, N., et al. LTE in the unlicensed spectrum: A novel coexistence analysis with WLAN systems. In Proceedings of the IEEE Global Communications Conference (GLOBECOM). Dec. 2014, p. 3489-3464. DOI: 10.1109/GLOBCOM.2014.7037343

[16] MA, Y., KUESTER, D. G., CODER, J., et al. A simulation study of the LTE interference on WiFi signal detection. In Proceedings of the URSI National Radio Science Meeting (URSI NRSM). Boulder (USA), 2016, p. 1-2. DOI: 10.1109/USNC-URSI-NRSM.2016.7436233

[17] MUKHERJEE, A., CHENG, J. F., FALAHATI, S., et al. System architecture and coexistence evaluation of licensed-assisted access LTE with IEEE 802.11. In Proceedings of the IEEE International Conference on Communications Workshop $(\mathrm{ICCW})$. London (United Kingdom), 2015, p. 2350-2355. DOI: 10.1109/ICCW.2015.7247532

[18] GIUPPONI, L., HENDERSON, T., BOJOVIC, B., et al. Simulating LTE and Wi-Fi Coexistence in Unlicensed Spectrum with ns-3. 12 pages. [Online] Cited 2016-07-25. Available at: www.arxiv.org/abs/1604.06826

[19] HUAWEI TECHNOLOGIES CO., LTD., CHINA. U-LTE: Unlicensed Spectrum Utilization of LTE (white paper). 20 pages. [Online] Cited 2015-05-15. Available at: http://www.huawei.com/

[20] IEEE COMPUTER SOCIETY STD., USA. 802.11n, Part 11: Wireless LAN Medium Access Control (MAC) and Physical Layer Specifications. 536 pages. [Online] Cited 2015-05-30. Available at: http://standards.ieee.org/getieee802/download/802.11n-2009.pdf

[21] CABAN, S., RUPP, M., MEHLFÜHRER, C., et al. Evaluation of HSDPA and LTE: From Testbed Measurements to System Level Performance. 1st ed. Wiley, 2011. ISBN: 9780470711927

[22] BRUENINGHAUS, K., ASTELY, D., SALZER, T., et al. Link performance models for system level simulations of broadband radio access systems. In Proceedings of the 16th IEEE International Symposium on Personal, Indoor and Mobile Radio Communications. Sep. 2005 p. 2306-2311. DOI: 10.1109/PIMRC.2005.1651855

[23] IEEE TGm. IEEE 802.16m Evaluation Methodology Document (EMD): Evaluation Methodology for P802.16m-Advanced Air Interface. 162 pages. [Online] Cited 2016-09-26. Available at: http://ieee802.org/16/tgm/docs/80216m-08_004r2.pdf 
[24] IKUNO, J. C., WRULICH, M., RUPP, M. System level simulation of LTE networks. In Proceedings of the 71st IEEE Vehicular Technology Conference (VTC 2010-Spring). May 2010, p. 1-5. DOI: 10.1109/VETECS.2010.5494007

[25] GLEISSNER, F., HANUS, S. Co-channel and adjacent channel interference measurement of UMTS and GSM/EDGE systems in $900 \mathrm{MHz}$ radio band. Radioengineering, 2008, vol. 17, no. 3, p. 74-80. ISSN: $1805-9600$

[26] MEHLFÜHRER, C., WRULICH, M., IKUNO, J. C., et al. Simulating the Long Term Evolution physical layer. In Proceedings of the 17th European Signal Processing Conference (EUSIPCO 2009). Glasgow, 2009, p. 1471-1478. ISBN: 978-161-7388-76-7

[27] VIENNA UNIVERSITY OF TECHNOLOGY, AUSTRIA. LTE Downlink Link Level Simulator. [Online] Cited 2015-0529. Available at: http://www.nt.tuwien.ac.at/research/mobilecommunications/vienna-lte-a-simulators/

[28] MILOS, J., HANUS, S. Performance analysis of PCFICH and PDCCH LTE control channels. Radioengineering, 2014, vol. 23, no. 1, p. 445-451. ISSN: $1805-9600$

[29] MILOS, J., HANUS, S. Analysis of LTE physical Hybrid-ARQ control channel. Advances in Electrical and Computer Engineering, 2014, vol. 14, no. 2, p. 97-100. DOI: 10.4316/AECE.2014.02016

[30] MILOS, J., POLAK, L., SLANINA, M., et al. Link-level simulator for WLAN networks. In Proceedings of the 1st International Workshop on Link- and System Level Simulations (IWSLS ${ }^{2}$ ). Vienna (Austria), 2016, p. 1-4.

[31] TRANTER, W. H. Principles of Communication Systems Simulation with Wireless Applications. Upper Saddle River (USA): Prentice Hall, 2004. ISBN: 0-13-494790-8

[32] MILOS, J., POLAK, L., SLANINA, M., et al. Measurement setup for evaluation the coexistence between LTE downlink and WLAN networks. In Proceedings of the 10th International Symposium on Communication Systems, Networks and Digital Signal Processing (CSNDSP). Prague (Czech Republic), 2016, p. 1-4.

[33] 3rd GENERATION PARTNERSHIP PROJECT. Evolved Universal Terrestrial Radio Access (E-UTRA) and Evolved Universal Terrestrial Radio Access Network (E-UTRAN); Overall Description; Stage 2. Ver. 8.12.0 TS 36.300. 147 pages. [Online] Cited 2015-06-01. Available at: www.3gpp.org/ftp/Specs/archive/36_series/36.300/363008c0.zip

[34] LTE-U Forum. LTE-U Forum: Coexistence Study for LTE-U SDL (technical paper V1.0). 52 pages. [Online] Cited 2016-07-26. Available at: www.lteuforum.org/documents.html

[35] KUDER, Z., MILOS, J., HANUS, S. Radio coexistence of major and upcoming wireless standards in the ISM bands. In Proceedings of the 26th IEEE International Conference Radioelektronika. Košice (Slovakia), 2016, p. 1-4. DOI: 10.1109/RADIOELEK.2016.7477426
[36] GOLDSMITH, A. Wireless Communications. 1st ed. London (UK): Cambridge University Press, 2005. ISBN: 0521837162

\section{About the Authors...}

Jiří MILOŠ was born in Uherské Hradiště, the Czech Republic in 1986. He received his M.Sc. and Ph.D. degrees at the Faculty of Electrical Engineering and Communications from the Brno University of Technology. His research interests are modeling, simulation and measurement of wireless and cellular communication technologies.

Ladislav POLÁK was born in Štúrovo, Slovakia in 1984. He received his M.Sc. degree in 2009 and Ph.D. degree in 2013, both in Electronics and Communication from the Brno University of Technology (BUT), the Czech Republic. Currently he is an assistant professor at the Department of Radio Electronic (DREL), BUT. His research interests are Digital Video Broadcasting (DVB) standards, wireless communication systems, simulation and measurement of the coexistence between wireless communication systems, signal processing, video image quality evaluation and design of subjective video quality methodologies. He has been an IEEE member since 2010.

Stanislav HANUS was born in Brno, the Czech Republic, in 1950. He received his M.Sc. and Ph.D. degrees from the Brno University of Technology. He is Professor at the Department of Radio Electronics, Faculty of Electrical Engineering and Communication in Brno. His research is concentrated on Mobile Communications and Television Technology.

Tomáš KRATOCHVíL was born in Brno, Czech Republic, in 1976. He received the M.Sc. degree in 1999, Ph.D. degree in 2006 and Assoc. Prof. position in 2009, all in Electronics and Communications from the Brno University of Technology. He is currently a full professor at the Department of Radio Electronics, Brno University of Technology. His research interests include digital television and audio broadcasting, its standardization and video and multimedia transmission including video image quality evaluation. He has been an IEEE member since 2001 and senior member since 2016. 